\title{
The analytical basis for the resonances and anti-resonances of loop antennas and meta- material ring resonators
}

Cite as: J. Appl. Phys. 112, 094911 (2012); https://doi.org/10.1063/1.4764104

Submitted: 27 July 2012 . Accepted: 09 October 2012 . Published Online: 12 November 2012

A. F. McKinley, T. P. White, I. S. Maksymov, and K. R. Catchpole

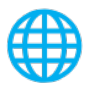

\section{ARTICLES YOU MAY BE INTERESTED IN}

Theory of the circular closed loop antenna in the terahertz, infrared, and optical regions Journal of Applied Physics 114, 044317 (2013); https://doi.org/10.1063/1.4816619

Theory of the Thin Circular Loop Antenna

Journal of Mathematical Physics 3, 1301 (1962); https://doi.org/10.1063/1.1703875

Probing the transition from an uncoupled to a strong near-field coupled regime between bright and dark mode resonators in metasurfaces

Applied Physics Letters 105, 081108 (2014); https://doi.org/10.1063/1.4893726

\section{ri AlluXa YOUROPTICAL COATING PARTNER}




\title{
The analytical basis for the resonances and anti-resonances of loop antennas and meta-material ring resonators
}

\author{
A. F. McKinley, ${ }^{1, \text { a) }}$ T. P. White, ${ }^{1}$ I. S. Maksymov, ${ }^{2}$ and K. R. Catchpole ${ }^{1}$ \\ ${ }^{1}$ Centre For Sustainable Energy Systems, College of Engineering and Computer Science, \\ The Australian National University, Engineering Bldg 32-Canberra, ACT 0200, Australia \\ ${ }^{2}$ Nonlinear Physics Centre, Research School of Physics and Engineering, The Australian National University, \\ Le Couteur Bldg 59-Canberra, ACT 0200, Australia
}

(Received 27 July 2012; accepted 9 October 2012; published online 12 November 2012)

\begin{abstract}
Interest in the electromagnetic properties of loop structures has surged with the recent appearance of split-ring resonator meta-materials (SRRs) and nano-antennas. Understanding the resonances, anti-resonances, and harmonics of these loops is key to understanding their response to a wide range of excitation wavelengths. We present the classical analytical solution for the input impedance of a loop structure with circumference on the order of the wavelength, and we show how to identify these resonances from the function. We transform the classical solution into a new RLC formulation and show that each natural mode of the loop can be represented as a series resonant circuit, such that the full response function can be resolved by placing all of these circuits in parallel. We show how this formulation applies to SRRs. (C) 2012 American Institute of Physics. [http://dx.doi.org/10.1063/1.4764104]
\end{abstract}

\section{INTRODUCTION}

Large loops, designed for the radio frequency (RF) regime, have received minimal attention over the past 50 years compared with other types of antennas, such as dipoles and cavities. However, the recent design of the split-ring resonator for the microwave (MW) region, ${ }^{1}$ for example, has shown that loops can play a vital role in new and interesting applications, such as cloaking. Small loop antennas are now being suggested for sensors, light energy directivity, and focusing. ${ }^{2-9}$

A number of recent papers have sought RLC circuit models for general plasmonic objects ${ }^{10-13}$ and for nanoantennas. ${ }^{14,15}$ Loops have also received some interest in this regard, because loops are relatively easy to build at the nanoscale and because RLC models clearly display resonances. ${ }^{16,17}$ For example, in designing his split-ring resonator meta-materials (SRR), Pendry ${ }^{1}$ focused on a calculation of its main resonance $\left(\omega_{0}\right)$, its permeability (that is, its inductance), capacitance, and quality factor (Q). Indeed, some authors have developed RLC circuit models that are intended to give all of the modal resonances. ${ }^{18,19}$ Consequently, these resonances are vital, whether one wants to design a nanostructure for meta-materials or for high frequency radiation.

Many of these recent models work well in the particular case for which the model was developed, but there is no general RLC model that captures the essential physical behavior of circular loops over the entire frequency range from RF through the optical region. In general, these specific models are too simple in that they rely on constant values for the elements used. In this paper, we transform a known physical model of the circular loop, developed for use in the RF region during the 1950s, into a form which emphasizes an RLC formulation. We show that only for extremely thin loops are the

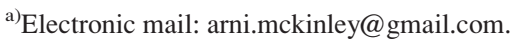

resulting $\mathrm{R}, \mathrm{L}$, and $\mathrm{C}$ functions constant over frequency. For thicker loops, which are currently the only kind of loops being fabricated in the MW, low THZ and optical frequencies, the RLC values must be replaced by functions of frequency. The ramifications of this are that the resonances, anti-resonances, bandwidths, and elemental RLC values for all harmonic modes of reasonably thick circular loops can be well predicted from the RF through low $\mathrm{THz}$ region.

In Sec. II, we describe the geometry of the circular loop used in this study, and we show how to identify its resonances and anti-resonances from its input impedance function. We also suggest the conditions for which a loop should be designed around a resonance or around an anti-resonance. In Sec. III, we show the results of the classical derivation of the input impedance function. In Sec. IV, we transform this result into one which identifies each mode as a series resonant RLC circuit, and we give functions for the R, L, and C of each mode, including the zero order mode. In Sec. V, we study the accuracy limits of the functions by making two comparisons with numerical simulations and with SRRs found in the literature. In Sec. VI, we discuss various details of our work, with emphasis on an intuitive understanding of the physics of circular loops.

\section{RESONANCES AND ANTI-RESONANCES}

Figure 1(a) shows the geometrical structure of the classical circular loop antenna, characterized by the loop radius, $b$, the wire radius, $a$, and a unit-less thickness measure, $\Omega=2 \ln$ $(2 \pi b / a)$. A delta function voltage source placed across an infinitesimal gap, $V \delta(\phi=0)$, drives the loop. The derivation of the loop's response to this driving function, given in Sec. III, imposes two restrictions: (1) the material is perfectly conducting and (2) $a^{2} \ll b^{2}$. The first limits the results to frequencies below $1 \mathrm{THz}$. The second limits the results to loops of thickness $\Omega>8$. The results are plotted against the independent 


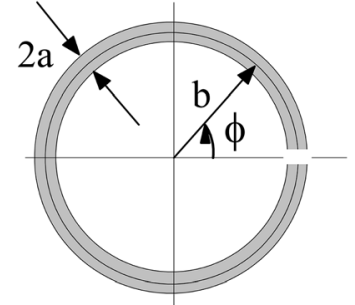

(a)

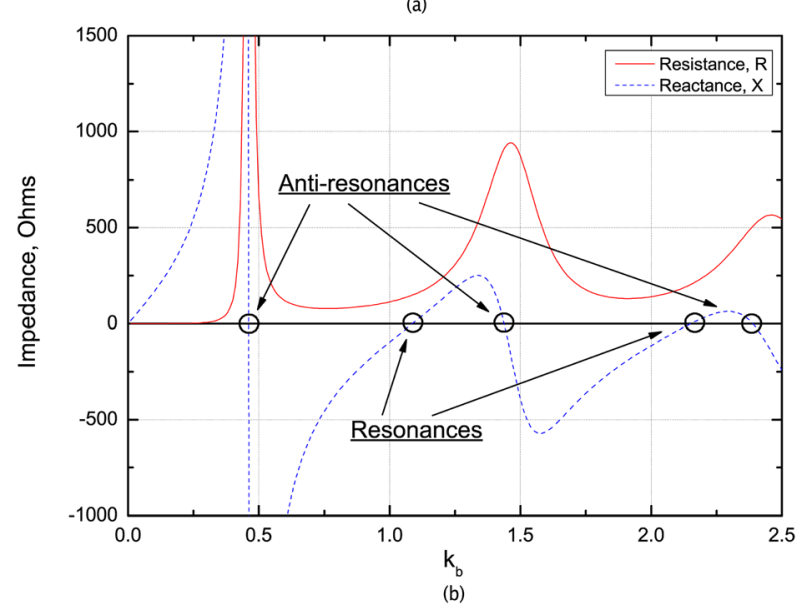

FIG. 1. (a) Geometry of the classical loop, showing the key variables. The gap is infinitesimal and supports a "delta-function" driving voltage. (b) Plot of the impedance for a loop of size $\Omega=12$, i.e., $b / a \approx 64$.

variable $k_{b}=2 \pi b / \lambda$, where $\lambda$ is the exciting wavelength. An increase in this value can be viewed in two ways: (1) as an increase in the size of the circumference given a constant excitation wavelength, or (2) as a decrease in the excitation wavelength given a constant circumference.

The response of the loop to the driving voltage in the RF region has been well known since the 1950 s. $^{20}$ Figure 1 shows the complex impedance function of a relatively thin loop $(\Omega=12)$ for $0<k_{b}<2.5$. The real part of the function is the resistance, $\mathrm{R}$; the imaginary part is the reactance, $\mathrm{X}$. The impedance function is important because it specifies the loop's resonant behavior. Resonances are of two types: true resonances, namely those frequencies where the current wave traveling around the circumference of the loop, reinforces itself; and anti-resonances, where the wave cancels itself exactly. At a true resonance, current flows freely in the loop and builds to a large quantity. At an anti-resonance, little current flows in the loop.

The resonated loop is interesting among resonant systems, because it wants to store energy in current flow and yet radiate energy at the same time. Consequently, resonated loops tend to have $Q s$ on the order of 3 to $8,{ }^{21}$ not very high and not very low. In fact, all of the harmonic resonances have $Q \mathrm{~s}$ in this range, indicating that the loop behaves the same at many frequencies simultaneously. This is particularly true of thin loops, as we shall discuss in Sec. VI.

On the other hand, the $Q$ of a loop at an anti-resonance is zero, because there is no energy stored. However, if a gap exists within the loop, then a high $\vec{E}$ field can appear across the gap, and energy will be stored within that field, just like it would be in a capacitance. As a result, an anti-resonance can also have a high quality factor. In fact, the $Q$ can be much higher than the $Q$ at a resonance, because little energy can escape the gap. We have no estimates for anti-resonance $Q$ s.

The proper way to find either type of resonance is to examine those points where the reactance goes to zero. These zerocrossings are marked by circles in Figure 1(b). At true resonances, the reactance changes from negative to positive (capacitive to inductive reactance). At anti-resonance, the reverse occurs. All true harmonic resonances occur near integer values of $k_{b}$, since this is where the wave is reinforced. The loop loses all reactance and appears to the source to be purely resistive; this resistance is, in fact, the radiation resistance of the loop at that resonance. On the other hand, all anti-resonances occur about half way between consecutive integer values of $k_{b}$. Again the loop has no reactance and appears to the source to be purely resistive, but this resistance is quite high, typically much larger than any radiation resistance at the resonances.

\section{THE CLASSICAL IMPEDANCE FUNCTION OF THE CIRCULAR LOOP}

The complete derivation of the input impedance of the circular loop was accomplished by Hallen, ${ }^{22}$ Storer, ${ }^{20}$ and $\mathrm{Wu}^{23}$ A delta function voltage, $V \delta(0)$, across an infinitesimal gap generates a current, which may be described by an infinite Fourier series of natural resonant modes.

$$
I(\phi)=\sum_{m=-\infty}^{\infty} I_{m} \exp (j m \phi)=\frac{V \delta(\phi)}{j \pi \xi_{0}}\left[\frac{1}{a_{0}}+2 \sum_{m=1}^{\infty} \frac{\cos (m \phi)}{a_{m}}\right]
$$

$\xi_{0}=377 \Omega$ is the impedance of free space. The input impedance at $\phi=0$ follows by definition:

$$
Z=\frac{j \pi \xi_{0}}{\frac{1}{a_{0}}+2 \sum_{m=1}^{\infty} \frac{1}{a_{m}}}
$$

This approach has been cited in all of the major antenna textbooks and in the literature since then; for example, Refs. 21 and 24-29. Under the assumption of a perfect electrical conductor, which limits the applicability of the derivation to frequencies below the low $\mathrm{THz}$ regime, and under the assumption that the wire radius is much smaller than the radius of the loop $\left(a^{2} \ll b^{2}\right)$, the coefficients are given by Storer $^{20}$

$$
a_{m}=a_{-m}=k_{b}\left(\frac{N_{m+1}+N_{m-1}}{2}\right)-\frac{m^{2}}{k_{b}} N_{m} .
$$

In solving a particular problem expressed by Storer, $\mathrm{Wu}^{23}$ was able to reduce Storer's recursive expression for $N_{m}$ to the regular expression

$$
\begin{aligned}
N_{m}= & N_{-m}=\frac{1}{\pi}\left[K_{0}\left(\frac{m a}{b}\right) I_{0}\left(\frac{m a}{b}\right)+C_{m}\right] \\
& -\frac{1}{2} \int_{0}^{2 k_{b}}\left[\Omega_{2 m}(x)+j J_{2 m}(x)\right] d x \text { for } m>1
\end{aligned}
$$

and $C_{m}=\ln (4 m)+\gamma-2 \sum_{k=0}^{m-1} 1 /(2 k+1) ; \gamma$ is Euler's constant, 0.5772 . The zero order term is 


$$
N_{0}=\frac{1}{\pi} \ln \left(\frac{8 b}{a}\right)-\frac{1}{2}\left[\int_{0}^{2 k_{b}}\left[\Omega_{0}(x)+j J_{0}(x)\right] d x\right] .
$$

This is now considered the final solution. ${ }^{30} \Omega_{m}(x)$ and $J_{m}(x)$ are the Lommel-Weber function and the Bessel function of the first kind, respectively. $I_{0}(x)=1+O\left(x^{2}\right) \approx 1$ and $K_{0}(x)=-(\ln (x / 2)+\gamma) I_{0}(x)+O\left(x^{2}\right) \approx-\ln (x / 2)-\gamma$ are modified Bessel functions of the first and second kind, respectively. ${ }^{31}$

Storer replaced the summation terms $m>5$ with an integral, ${ }^{20}$ which he evaluated as

$$
\Psi=\frac{2 \pi}{\ln \left(\frac{n_{o}}{4.5}\right)} \frac{k_{b}}{4.5}\left[J+\frac{1}{3}\left(\frac{k_{b}}{4.5}\right)^{2}\right],
$$

$J=\{0.47,0.9,1.25,1.4,1.4\} \quad$ for $\quad \Omega=8,9,10,11,12$; where $n_{0}=2 b / a \exp (-\gamma)$.

This substitution significantly speeds the calculation. The series now appears as

$$
Z=\frac{j \pi \xi_{0}}{\frac{1}{a_{0}}+2 \sum_{m=1}^{4} \frac{1}{a_{m}}-\Psi} .
$$

\section{THE RLC REPRESENTATION OF THE CIRCULAR LOOP}

We now cast the impedance function into an RLC form by applying the transformations, $Z_{0} \equiv j \pi \xi_{o} a_{0}$ and $Z_{m} \equiv j \pi \xi_{o} a_{m} / 2$. The classical impedance function Eq. (2) then becomes

$$
Z=\frac{1}{\frac{1}{Z_{0}}+\sum_{m=1}^{\infty} \frac{1}{Z_{m}}}
$$

This is evidently a parallel system of impedances as viewed from the gap. Figure 2 shows a representation of Eq. (6). Using Eq. (3) and Eq. (4), and the definitions

$$
\begin{aligned}
R_{m} & \equiv m \xi_{o}\left[k_{b} r_{m} / m-m /\left(k_{b} g_{m}\right)\right] \\
L_{m} & \equiv \mu_{o} b l_{\mu m} \\
C_{m} & \equiv \epsilon_{o} b l_{\epsilon m} / m^{2}
\end{aligned}
$$

the modal impedance becomes

$$
\begin{aligned}
Z_{m} & =R_{m}+j\left(\omega L_{m}-\frac{1}{\omega C_{m}}\right) \\
& =m \xi_{o}\left[\left(\frac{k_{b} r_{m}}{m}-\frac{m}{k_{b} g_{m}}\right)+j\left(\frac{k_{b} l_{\mu m}}{m}-\frac{m}{k_{b} l_{\epsilon m}}\right)\right],
\end{aligned}
$$

where $r_{m}, g_{m}, l_{\mu m}$, and $l_{\epsilon m}$ are unit-less. These definitions lead to

$$
r_{m}=\left\{\begin{array}{ccc}
\frac{\pi}{8} \int_{0}^{2 k_{b}}\left(J_{2(m+1)}(x)+J_{2(m-1)}(x)\right) d x & \text { if } & m>0, \\
\frac{\pi}{2} \int_{0}^{2 k_{b}} J_{2}(x) d x & \text { if } & m=0 .
\end{array}\right.
$$

$$
g_{m}=\left\{\begin{array}{clc}
1 /\left[\frac{\pi}{4} \int_{0}^{2 k_{b}} J_{2 m}(x) d x\right] & \text { if } & m>0 \\
\infty & \text { if } & m=0 .
\end{array}\right.
$$

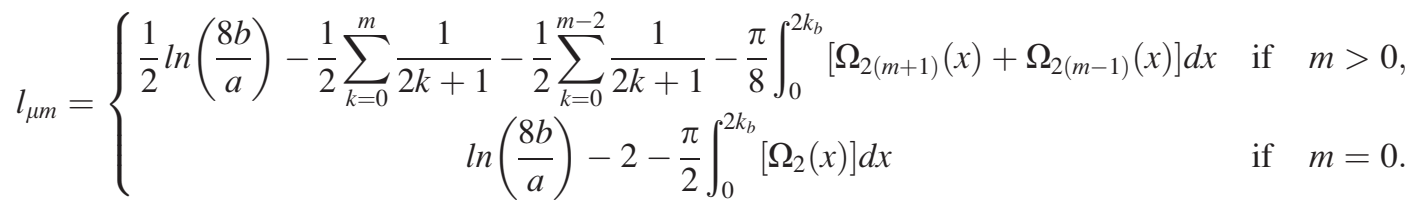

$$
l_{\epsilon m}=\left\{\begin{array}{cl}
2 /\left[\ln \left(\frac{8 b}{a}\right)-2 \sum_{k=0}^{m-1} \frac{1}{2 k+1}-\frac{\pi}{2} \int_{0}^{2 k_{b}}\left[\Omega_{2 m}(x)\right] d x\right] & \text { if } \quad m>0 \\
\infty & \text { if } \quad m=0
\end{array}\right.
$$

$\mu_{0}$ and $\epsilon_{0}$ are the permeability and permittivity of free space. The transform to $k$-space is $\omega=k_{b} c / b$.

The individual $R_{m}, L_{m}$, and $C_{m}$ elements are functions of $k_{b}$, rather than constants, as in standard resonant circuit theory. As an example, we can use the standard definition for the resonant frequency, $\omega_{m}=1 /\left(L_{m} C_{m}\right)^{1 / 2}$, and introduce the term "modal balanced reactance" for $X_{b m}=\left(L_{m} / C_{m}\right)^{1 / 2}$, to find that

$$
\begin{aligned}
Z_{m} & =R_{m}+j X_{b m}\left(\frac{\omega}{\omega_{m}}-\frac{\omega_{m}}{\omega}\right) \\
& =R_{m}+j X_{b m}\left(\frac{k_{b}}{k_{b m}}-\frac{k_{b m}}{k_{b}}\right) .
\end{aligned}
$$

Figure 3 show the natural resonances for mode $2(m=2)$ for an extremely thin loop and for a thick loop, as given by 


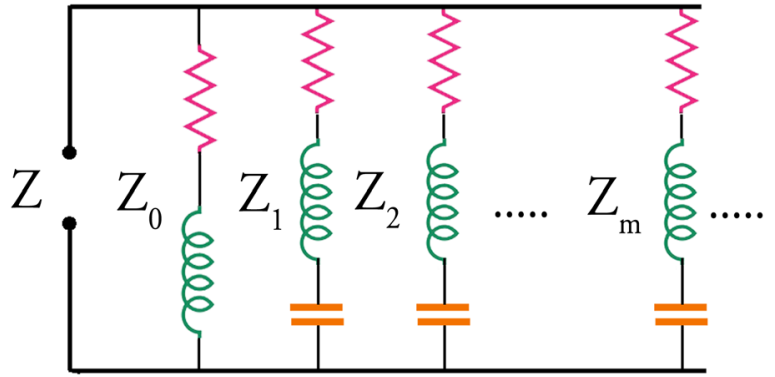

FIG. 2. The proper RLC model of a circular loop antenna in standard form.

$1 / Z_{2} \equiv 1 /\left(j \pi \xi_{o} a_{m} / 2\right)$. The modal resonances of an infinitesimally thin loop have traveling waves that have wavelengths, which fit the circumference exactly. This produces a very narrow bandwidth and, therefore, a high $\mathrm{Q}$, because only a few wavelengths can participate in the resonance. In this case alone, can constant values be found for the series R, L, and $\mathrm{C}$ of each mode, as the comparison in (a) shows between the theoretical natural resonance and one given by an RLC model. Thick loops, on the other hand, present a wide choice of path length due to wire thickness, thereby broadening the
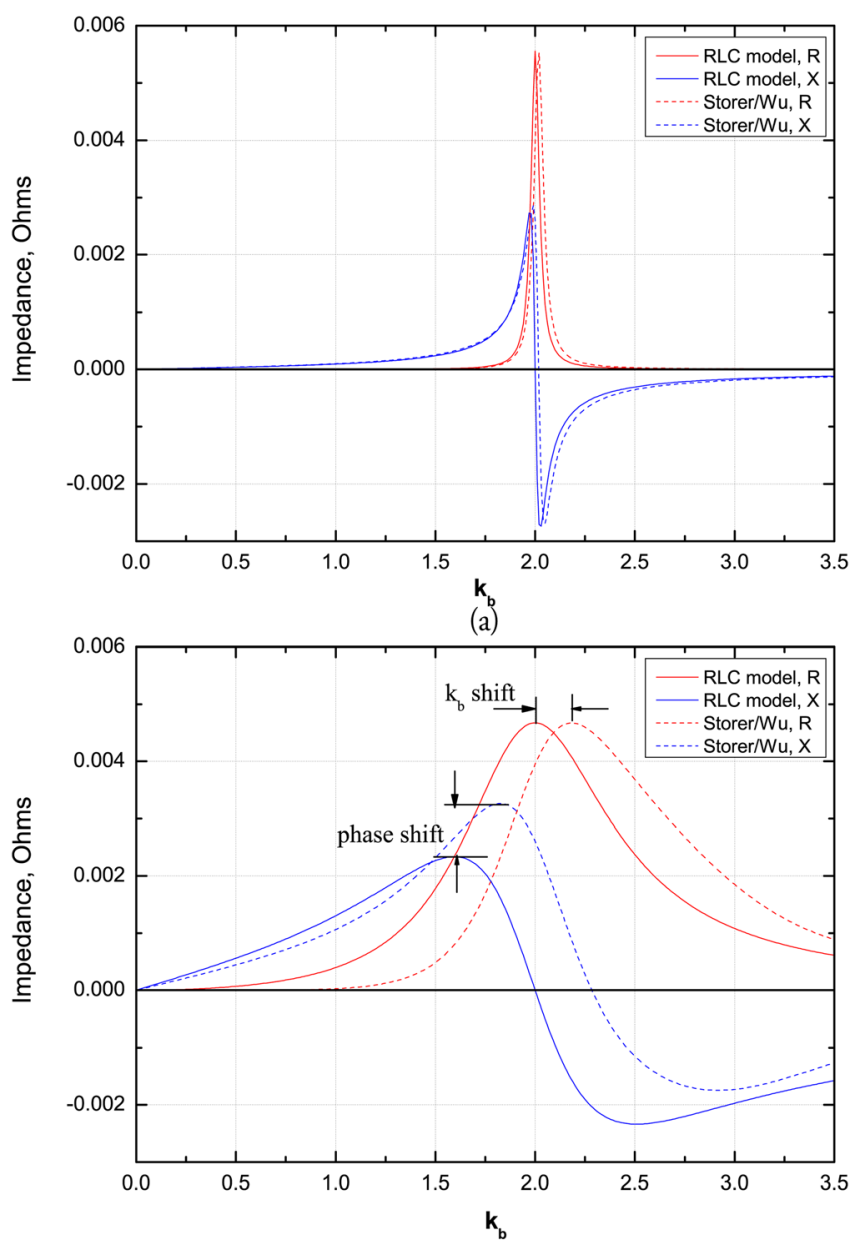

(b)

FIG. 3. Mode 2 resonance for (a) an extremely thin loop $(\Omega=40)$, comparing Storer/Wu's theory, $1 / Z_{2} \equiv 1 /\left(j \pi \xi_{o} a_{m} / 2\right)$, with an RLC model, Eq. (10), using constant values, $R=360 \mathrm{ohms}, X_{b}=14400 \mathrm{ohms}$; and for (b) a thicker loop $(\Omega=8)$ with $R=428 \mathrm{ohms}, X_{b}=1198 \mathrm{ohms}$. resonance, and lowering the $\mathrm{Q}$ value. Moreover, thicker loops have fewer harmonic resonances than do thinner loops.

Two principal discrepancies appear in Figure 3: a shift in the resonance away from $k_{b m}=m$ and a shift upward in the reactance (a phase shift). These shifts must be explained by theory and are done so by taking $\mathrm{R}, \mathrm{L}$, and $\mathrm{C}$ to be functions of $k_{b}$; that is, $R_{m}=R\left(k_{b}\right), L_{m}=L\left(k_{b}\right)$ and $C_{m}=C\left(k_{b}\right)$. Specifying these functions by Eq. (7) is the key result of this paper. We thus obtain an RLC model of the circular loop, in which the modes are treated as series RLC circuits with varying values, in parallel with each other and with a zero order mode, as illustrated in Figure 2.

As another example of the non-constancy of R, L, and $\mathrm{C}$, note that the modal resonant frequency, $\omega_{m}$, is not a constant value, but varies with frequency. The resonance function simplifies to $k_{b m}=m /\left(l_{\mu m} l_{\epsilon m}\right)^{1 / 2}$. The rolling curves shown in Figure 4 show the variation in $k_{b}$ for the first four modes. When $k_{b}=k_{b m}$, the loop resonates, hence the intersection of the line $y=k_{b}$ with the function $k_{b 1}$ gives the resonance of the first mode, and so forth with increasing $m$. These resonances, with associated values of the unit-less functions, are given in Table I. When referring to a value that occurs at a modal resonance, a tilde above the symbol will be used henceforth; for example, $\tilde{R}_{2}$ refers to the resistance of the loop at the modal resonance, $\tilde{k}_{b 2}$.

With these values, Eq. (7) can be calculated for each mode of a given loop, with radius $b$. It is important to note that the inductance and capacitance depend directly on the loop radius, but the resistance does not. Moreover, this resistance is not due to losses in the wire, since the model assumes perfectly conducting material. The resistance must, therefore, refer to radiation losses.

The zero order term consists of a resistance and an inductance in series,

$$
\begin{aligned}
Z_{0} & =R_{0}+j \omega L_{0}=\xi_{o} k_{b} r_{0}+j \omega \mu_{o} b l_{\mu 0} \\
& =\xi_{o} k_{b}\left[r_{o}+j l_{\mu 0}\right],
\end{aligned}
$$

where the terms are given by Eq. (9). Figure 5 shows the real and imaginary parts plotted over the range $0<k_{b}<2.5$ for $\Omega=12$. Again, the elements vary with $k_{b}$ and indeed the

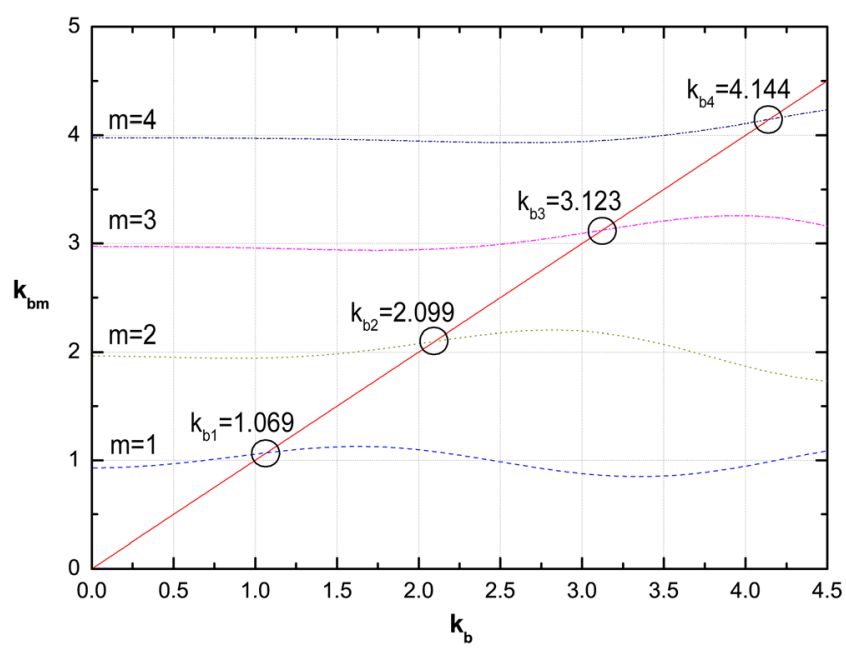

FIG. 4. The resonance functions, $k_{b m}$, for the first four modes, $\Omega=12$. 


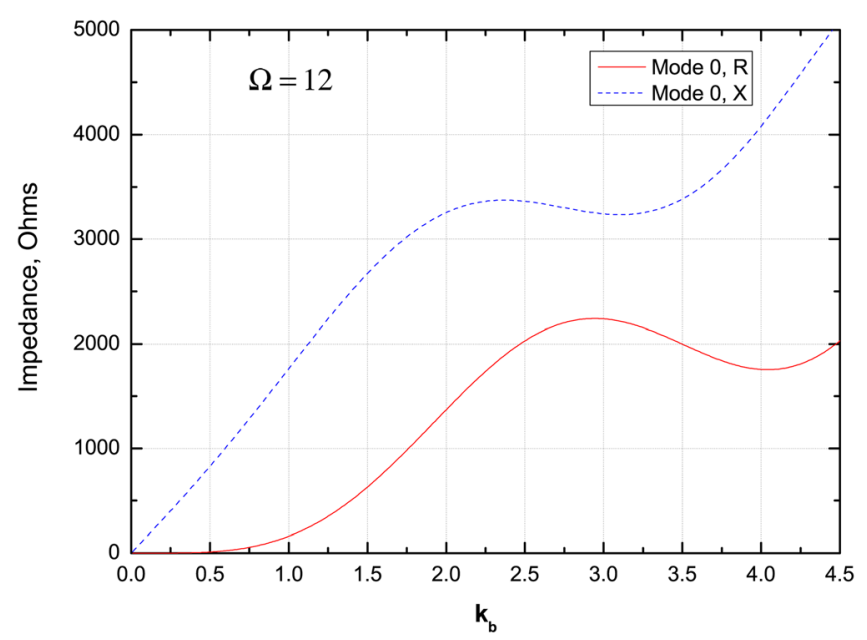

FIG. 5. The zero order resistance and reactance, $\xi_{0} k_{b} r_{o}$ and $\xi_{0} k_{b} l_{\mu 0} ; \Omega=12$.

magnitude of the zero order mode plays a crucial role in the full summation, as shown in the Table I.

In summary, every circular loop has an infinite number of resonant modes. However, it is the sum of these modes that yields the full impedance behavior, and the sum always converges to a more limited number of resonances. Indeed, as Figure 1 indicates, the zero crossings of the imaginary component tend to die off as the excitation frequency increases. For example, the resonances and anti-resonances of the first four natural modes of a loop with $\Omega=12$ are given in Table I under the heading $\tilde{k}_{b m}$. The first two resonances and the first two anti-resonances of the full impedance function itself are given in Table II. Notice that the values in these two tables are different and that all resonances and anti-resonances (except the first), disappear for loops of thickness $\Omega \leq 8$.

\section{COMPARISONS WITH SIMULATIONS AND REPORTS IN THE LITERATURE}

In order to show (a) the accuracy of the classically derived impedance function and (b) the power of this analytical method in identifying the resonances and anti-resonances
TABLE II. The first two resonances and anti-resonances for loops of thicknesses $\Omega=8$ through 12 .

\begin{tabular}{|c|c|c|c|c|}
\hline \multirow[b]{2}{*}{$\Omega$} & \multicolumn{2}{|c|}{ Anti-Resonances } & \multicolumn{2}{|c|}{ Resonances } \\
\hline & $k_{b}$ & $R, \mathrm{ohms}$ & $k_{b}$ & $R$, ohms \\
\hline \multirow[t]{2}{*}{12} & .460 & 28,620 & 1.087 & 148 \\
\hline & 1.438 & 912 & 2.151 & 203 \\
\hline \multirow[t]{2}{*}{10} & .452 & 18,030 & 1.150 & 165 \\
\hline & 1.39 & 423 & $\ldots$ & $\ldots$ \\
\hline \multirow[t]{2}{*}{8} & .455 & 8,601 & $\ldots$ & $\ldots$ \\
\hline & $\ldots$ & $\ldots$ & $\ldots$ & \\
\hline
\end{tabular}

of the circular loop, we perform two types of comparisons: (1) a comparison of the impedance functions of various circular loops as given by the RLC functions in Eq. (8), and as given by numerical simulations; (2) a comparison of the resonances of two SRRs as given by their impedance functions from numerical simulations and as given in the literature.

\section{A. Methodology}

For the first comparison, we simulate three loops in free space using CST's MICROWAVE STUDIO ${ }^{\text {TM }}$ (MWS). ${ }^{32}$ MWS is a $3 \mathrm{D}$, finite-element simulation tool applicable from RF through the optical region. We approximate the "infinitesimal gap" used in the analytical theory with a small gap at $\phi=0$, across which we place a power port to inject energy. In a real structure, the gap would introduce some capacitance that is not in the theory and would skew the resulting impedance function. Therefore, we remove the gap capacitance analytically in post-processing calculations, using a method suggested by Locatelli. ${ }^{2}$ The gap capacitance is calculated using $C_{g}=\epsilon_{0} A^{2} / g$, where $\epsilon_{0}$ is the permittivity of free space, $A$ is the cross-sectional area of the wire, and $g$ is the width of the gap.

For all simulation runs, the following guides are used:

Perfect electric conductors are used at all frequencies. The antenna sits in free space in the XY plane with the gap at $(0,-b, 0)$. Two symmetry planes are set: the $\mathrm{YZ}$ plane has

TABLE I. Key values at the modal resonances for $\Omega=12$.

\begin{tabular}{|c|c|c|c|c|c|c|c|c|c|c|c|c|c|}
\hline$\Omega=12$ & mode, $m$ & $\tilde{k}_{b m}$ & $\tilde{r_{0}}$ & $\tilde{R}_{0}$ & $\tilde{l_{\mu 0}}$ & $\tilde{X}_{L 0}$ & $\tilde{r}_{m}$ & $\tilde{g}_{m}$ & $\tilde{R}_{m}$ & $\tilde{l}_{\mu m}$ & $\tilde{l}_{\epsilon m}$ & $\tilde{X}_{b m}$ & $\tilde{X}_{b m} / \tilde{R}_{m}$ \\
\hline & 1 & 1.069 & .5075 & 204 & 4.70 & 1895 & .5776 & 3.9408 & 143.3 & 2.06 & .425 & 829 & 5.8 \\
\hline & 2 & 2.099 & 1.922 & 1521 & 4.19 & 3312 & .4976 & 3.5865 & 193.4 & 1.93 & .471 & 1526 & 7.9 \\
\hline & 3 & 3.123 & 1.876 & 2209 & 2.75 & 3234 & .4619 & 3.4429 & 228.3 & 1.84 & .502 & 2163 & 9.5 \\
\hline & 4 & 4.144 & 1.131 & 1767 & 2.79 & 4361 & .4414 & 3.3565 & 256.0 & 1.77 & .527 & 2759 & 10.8 \\
\hline \multirow[t]{5}{*}{$\Omega=10$} & mode, $m$ & $\tilde{k}_{b m}$ & $\tilde{r_{0}}$ & $\tilde{R}_{0}$ & $\tilde{l_{\mu 0}}$ & $\tilde{X}_{L 0}$ & $\tilde{r}_{m}$ & $\tilde{g}_{m}$ & $\tilde{R}_{m}$ & $\tilde{l}_{\mu m}$ & $\tilde{l}_{\epsilon m}$ & $\tilde{X}_{b m}$ & $\tilde{X}_{b m} / \tilde{R}_{m}$ \\
\hline & 1 & 1.096 & .540 & 223 & 3.712 & 1533 & .5813 & 3.701 & 147 & 1.544 & .539 & 638 & 4.3 \\
\hline & 2 & 2.147 & 1.967 & 1592 & 3.118 & 2523 & .5112 & 3.304 & 201 & 1.417 & .612 & 1147 & 5.7 \\
\hline & 3 & 3.190 & 1.815 & 2183 & 1.694 & 2038 & .4821 & 3.125 & 240 & 1.327 & .666 & 1596 & 6.7 \\
\hline & 4 & 4.230 & 1.128 & 1799 & 1.845 & 2943 & .4674 & 3.004 & 271 & 1.258 & .711 & 2007 & 7.4 \\
\hline \multirow[t]{5}{*}{$\Omega=8$} & mode, $m$ & $\tilde{k}_{b m}$ & $\tilde{r_{0}}$ & $\tilde{R}_{0}$ & $\tilde{l_{\mu 0}}$ & $\tilde{X}_{L 0}$ & $\tilde{r}_{m}$ & $\tilde{g}_{m}$ & $\tilde{R}_{m}$ & $\tilde{l}_{\mu m}$ & $\tilde{l}_{\epsilon m}$ & $\tilde{X}_{b m}$ & $\tilde{X}_{b m} / \tilde{R}_{m}$ \\
\hline & 1 & 1.162 & .625 & 274 & 2.732 & 1197 & .5881 & 3.202 & 156 & 1.011 & .732 & 443 & 2.8 \\
\hline & 2 & 2.284 & 2.070 & 1783 & 1.911 & 1646 & .5455 & 2.661 & 222 & .881 & .870 & 759 & 3.4 \\
\hline & 3 & 3.420 & 1.591 & 2052 & .5803 & 748 & .5448 & 2.316 & 274 & .786 & .979 & 1013 & 3.7 \\
\hline & 4 & 4.580 & 1.237 & 2136 & 1.035 & 1788 & .5630 & 2.027 & 322 & .708 & 1.077 & 1223 & 3.8 \\
\hline
\end{tabular}


the tangential component of $\vec{E}=0$ (i.e., $\vec{E}_{t}=0$ ) and the $\mathrm{XY}$ plane $\vec{H}_{t}=0$. The gap for the circular loop is kept at $0.03 b$ and its capacitance removed from the data in postprocessing. A discrete power port, placed across the gap, provides excitation, with a source power of 1 watt and variable port resistance, $R_{p}$. The design frequency is defined: $f_{d}=c /(2 \pi b)$, where $c$ is the speed of light. The frequency range is set: $<.1 f_{d}$ to $2.5 f_{d}$. The horizontal axis measure, $k_{b}$, is calculated in post-processing by dividing this range by $f_{d}$. Post-processing calculates the real and imaginary parts of the impedance, $Z$, and the reflected power, $S_{11}^{2} . R_{p}$ is at first arbitrary, but is iteratively replaced until $S_{11}^{2}$ reaches minimum. At this value, $R_{p}$ equals $\operatorname{Re}(Z)$ and maximum power flows from the port into the antenna. If the antenna resonates, the minimum occurs very close to $\operatorname{Im}(Z)=0$.

\section{B. Results}

Figure 6 compares MWS simulations ${ }^{33}$ of the impedance with theory for three loops. Theory matches the simulation well throughout the $k_{b}$ range for the thin loop, shown in (a). As the loop thickens in (b), and then in (c), the match becomes less accurate. This is expected, given the assumptions of the derivation, given in Sec. II.

It was noted in Sec. II that the individual modes of thin loops have very sharp, high $Q$, narrow resonances, and that they do not overlap in the full impedance summation. The effects of that are steep zero-crossings at the resonances very near $k_{b}=m$, which can be seen in Figure 6(a). Since the modal resonances of thick loops have lower $Q$ and are broader, the zero-crossings are less steep and $k_{b m}>m$. If the loop is thick enough, the resonances disappear entirely.

For a second test, we present two open-ringed structures studied by Zhou and Chui. ${ }^{19}$ In developing their analytical equations, they used the "quasi-static approximation (QSA)" of Maxwell's equations, an assumption specifically for structures that are small compared with the exciting wavelength. The assumption makes their analysis inherently weaker than that used by Storer and Wu when applied to large rings. Both rings have a gap of angular width $\pi / 40$, but our analysis is of rings without gaps, so a comparison between our resonances and theirs must take that into account. Figure 7 (a) shows a simulation of the first ring without the gap; we find an excellent match with our calculated impedance. Fig. 7(b) shows a simulation with the gap. Notice that the zero-crossings are slightly different than those in Fig. 7(a). Zhou and Chui's calculated resonances are compared with the zero-crossings. They call their minima at $0.5,1.42$, and 2.48 "odd-numbered resonances;" these correspond to our anti-resonances. Their "even-numbered resonances" near 0.96 and 2.0 correspond to our resonances of 1.05 and 2.07. We can attribute the 6\%-10\% difference only to their QSA assumption.

Zhou and Chui's second ring has $b=4 \mathrm{~mm}$ and $a=0.1 \mathrm{~mm}$ (our $\Omega=11.0$ ). They present the transmittance of a $2 \mathrm{D}$ planar structure, in which the $x y$ plane is tiled with these rings, using a lattice constant $16 \mathrm{~mm}$ in both directions. In Figure 8, we show their calculated and simulated results superimposed on the impedance resulting from our simula-
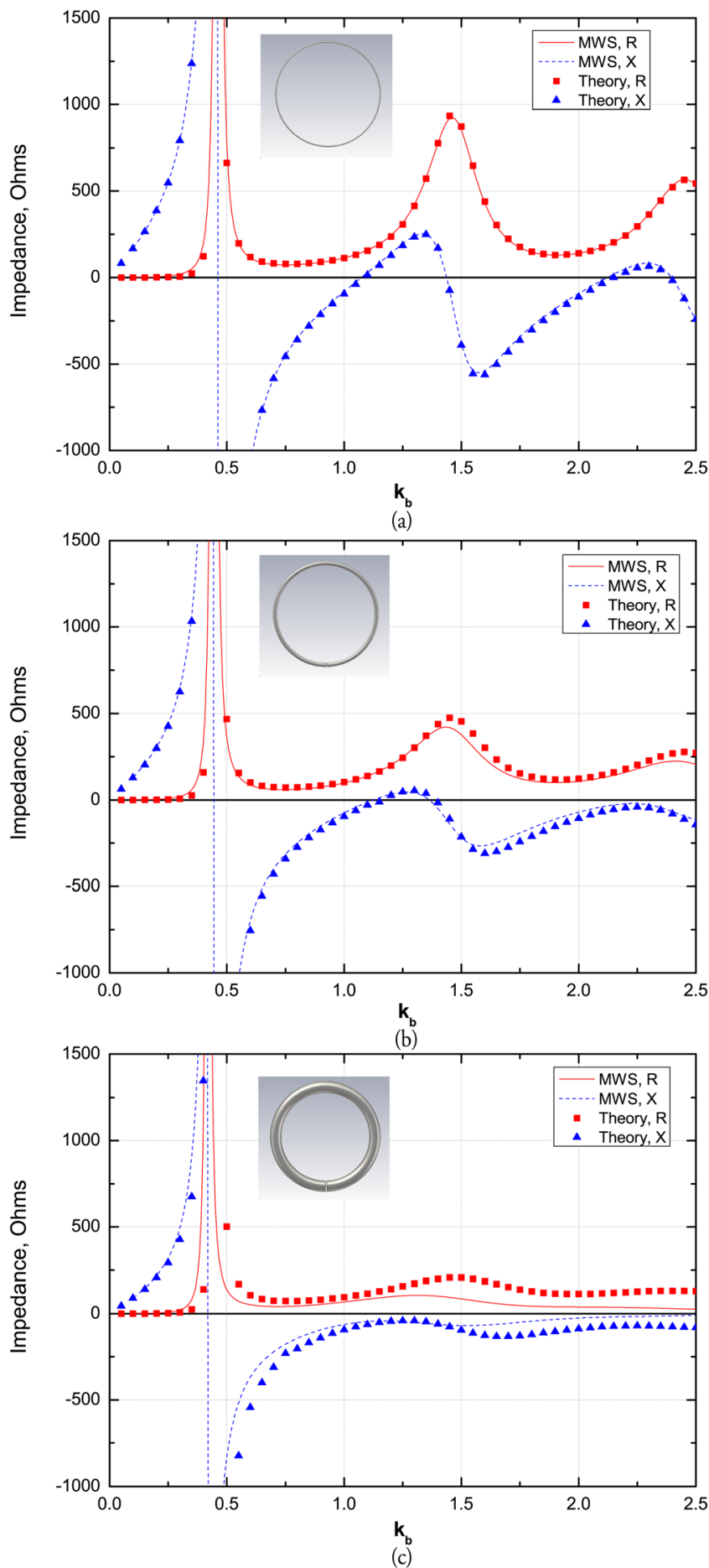

FIG. 6. The MWS simulation of a loop, with thickness $\Omega=$ (a) 12 , (b) 10 , and (c) 8. The analytical model, Eqs. (7) to (9), matches simulation results less and less as the loop becomes thicker, due to assumptions of the derivation. Note the steep zero-crossings at the true resonances for the thin loops, which indicate higher $Q s$ there.

tion. Note that while our anti-resonances closely match their odd-numbered resonances, our true resonance (1.11) falls midway between their calculated $(\approx .95)$ and simulated (1.19) even-numbered resonance. The difference between their calculated and simulated results is about $20 \%$. The difference may be due to the simulation of an array of rings vs the single ring itself. Figures in their papers show small structures near 2.0 and 2.4 , but they do not claim these as 


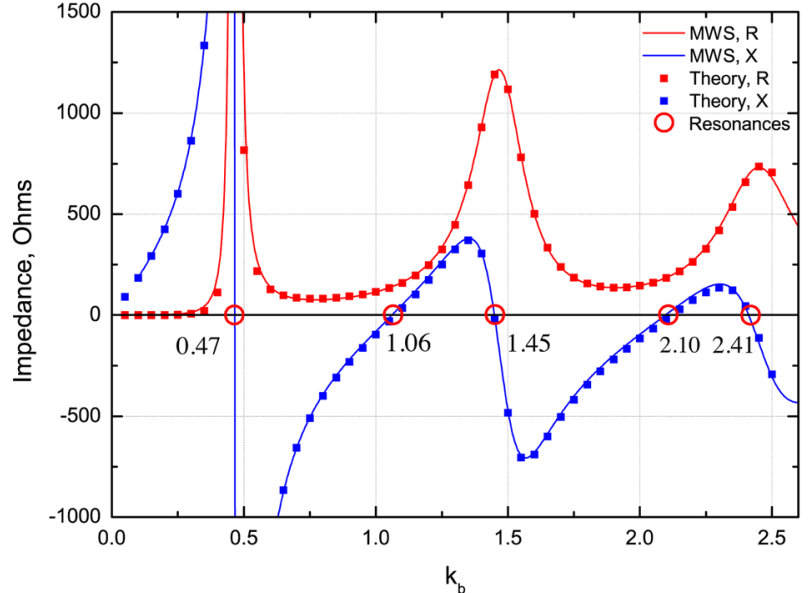

(a)

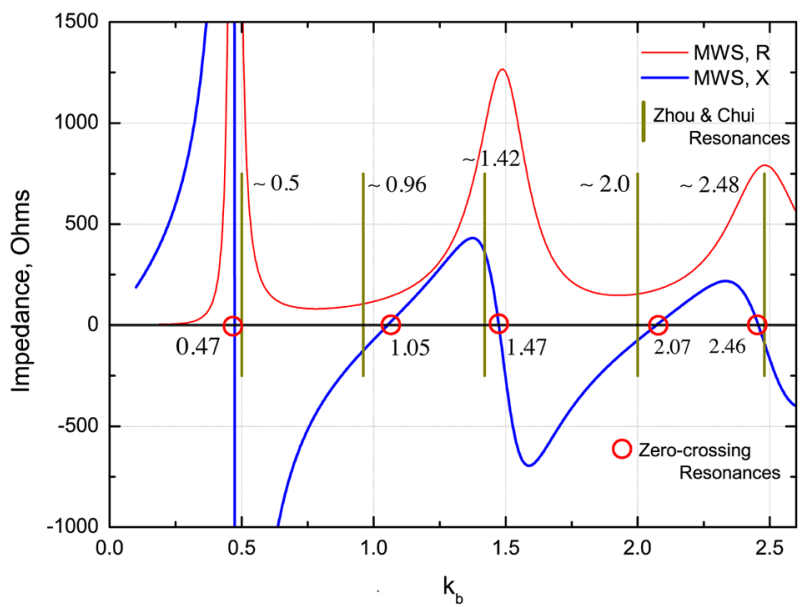

(b)

FIG. 7. Zhou and Chui's ${ }^{19}$ first ring $(\Omega=12.886)$ (a) without and (b) with the gap (size $=\pi / 40)$. Our resonances are slightly different, depending on whether the gap is present. (a) Compares our calculated impedance with simulated impedances. (b) Compares the zero-crossings of the simulated impedance with Zhou and Chui's calculated resonances.

resonances. Indeed they are not, since our theory shows no zero-crossings in this region.

\section{DISCUSSION}

Large circular loops evidently change their distributed resistance, inductance, and capacitance with frequency in a complicated way.

(1) At low frequencies, where the circumference is small with respect to the imposed wavelength, the loop looks inductive, with value $L_{0}=\mu_{0} b(\ln (8 b / a-2))$, a well known
result. ${ }^{29}$

(2) As the frequency rises, the wavelength approaches the length of the circumference. Naively, one would expect the traveling wave to reach the gap in phase with itself at every $k_{b}=m$ but this does not occur. This can be thought of, perhaps, as a slowing of the wave, but the definition $k_{b}=2 \pi b / \lambda$ has been used throughout, which implicitly assumes no slowing. Rather, four other reasons can explain the offsets: (a) the asymmetry between $l_{\mu m}$ and $l_{\epsilon m}$ defined in Eq. (9), (b) the influence of a strong zero term on all of the resonant modes, (c) the radius of the wire (a thicker wire has a greater variation in circular path-length for the traveling

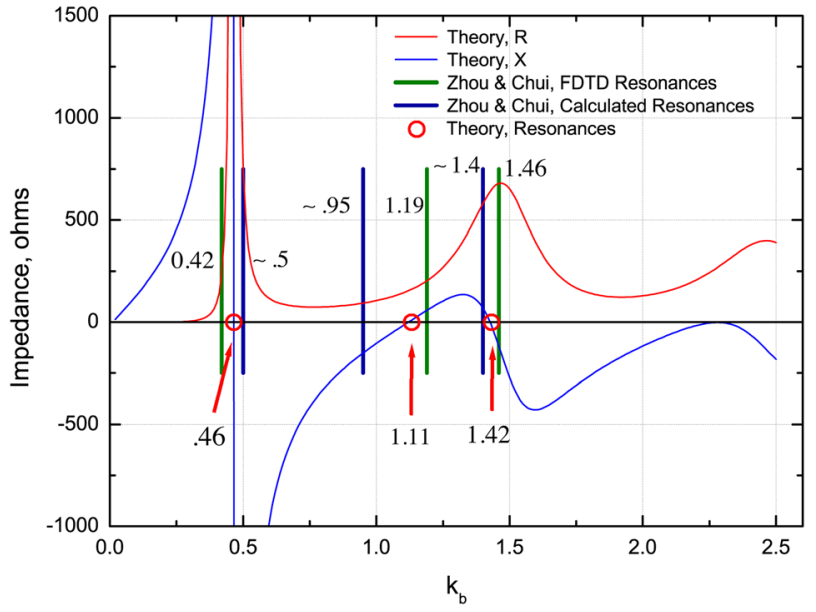

FIG. 8. Zhou and Chui's second ring, example Ref. 19 of size $\Omega=11.0$. Superimposed are our resonances and anti-resonances, their calculations of the resonances, and the resonances from their FDTD simulation of a $2 \mathrm{D}$ array using these rings.

wave than does a thinner wire), and (d) the way in which all modes affect every other mode, as discussed in Sec. II. In other words, at any given frequency, the loop presents itself, only approximately, as a series resistor and inductor in parallel with a series resistor, inductor, and capacitor. The result is a loop resonance that occurs at a frequency different than $k_{b}=m$; and indeed, at a slightly different place than the corresponding mode resonance itself.

In other words, the explanations for the $k_{b}$ shift are (a) none of the three unit-less functions are constant with $k_{b}$, and (b) an asymmetry exists between the functions $l_{\mu m}$ and $l_{\epsilon m}$. The differences between the summations in the latter two functions are slight, except for the first mode, $m=1$. Therefore, the asymmetry is due primarily to the Bessel integrals. These are enough to cause the shift in $k_{b}$ away from integer values. The phase shift discrepancy is due to the asymmetry between the real and imaginary parts of Eq. (8). Since it is impossible to assign constant values to $r_{m}, g_{m}, l_{\mu m}$, and $l_{\epsilon m}$, every mode affects every other mode, and for thick loops no single mode can be ascertained by looking at the total impedance.

(3) Roughly midway between the resonances, the traveling wave reaches the gap out of phase with itself exactly. Incoming power reflects back to the source strongly and the loop looks very resistive; hence peaks appear in the real part of the impedance. These are the anti-resonances. At these anti-resonances, the loop switches response from looking strongly inductive to strongly capacitive.

(4) On resonance, the imaginary portion of the impedance goes to zero, leaving the loop looking purely resistive. This is essentially the radiation resistance of the loop for that mode. The radiation resistance is not directly dependent on the loop radius, but rather on the ratio, $k_{b}$. At this point, the power reflected from the loop back toward the power source across the gap, reaches its lowest point. If there is a resonance, the reflected power goes to zero; if not, it reaches a minimum above zero.

(5) In the aggregate, the spectral response of the loop corresponds to that of a set of series resonant circuit elements in parallel, as illustrated in Figure 2 where the elements vary 
with frequency according to Eq. (9). The low frequency term is always present and has a strong influence over the behavior of the loop. The other modes act like RLC series resonant circuits. When the frequency is close to a mode resonance, the reactance for that mode gets close to 0 , and the series looks resistive; when the frequency is far away from the mode's resonance, the reactance looks like an open circuit. The other modes however affect the resonance of any other given mode, due to spectral broadening of each mode, as noted in Sec. II. Moreover, the zero-term impedance has a significant role in the overall impedance. Both the resistance and the reactance of this term have large values at each modal resonance.

(6) Figure 6 shows that the impedance function changes dramatically as the loop wire thickens. If a loop is extremely thin, the current wave traveling along the circumference finds only one path it can follow. In this case, the natural mode is very narrow, as shown in Figure 3(a). Notice that the tails of both the resistance and the reactance for this mode 2 of an $\Omega=40$ loop have small values at $k_{b}=1$ and $k_{b}=3$; that is, the modes do not interfere with each other and hence the full reactance function has steep zerocrossings very near all of the $k_{b}=m$. The $Q$ of this mode, as measured by the central resonance divided by the bandwidth, is quite high.

In a thicker loop, however, the circumferential path length is less clearly defined; the wavelength can fit a range of nearby paths, and hence a broadening of the resonant peak occurs, as in Figure 3(b). The $Q$ is lower and the slope of the zero-crossing of the full impedance curve at this resonance is small. Notice that the tails of the resonant curve for this mode overlap other modes. Broadened modes carry significant values at the other modes, and this tends to reduce the effectiveness of the overall loop response.

Consequently, in the RF region, where the emphasis has always been on communication and where radiating on multiple frequencies is undesirable, a trade-off exists in the choice between higher $\mathrm{Q}$, narrow band, thin loops with several harmonics and lower Q, broadband, thick loops with no harmonics. This tradeoff is one of the reasons that circular loops have not been useful in the RF region. The choice is not as critical at higher frequencies and smaller dimensions, where the research has so far focused on single frequency, thick loop, meta-material structures for cloaking and optical needs, rather than for communications.

\section{CONCLUSION}

We provide a set of equations describing an electrical RLC circuit model for thin and somewhat thick circular loops, applicable from the RF through low $\mathrm{THz}$ frequencies. The model provides all of the natural harmonic modes with their individual RLC representations. The full impedance function generated by the model provides the first resonance, first anti-resonance, and all of their harmonics.

In the RF, MW, THz, and soon in optical, regimes, loop antennas are used to control the storage, radiation, and focusing of energy. The key to such control is understanding the resonances and anti-resonances. Our method of finding first the impedance function of the loop and then identifying these resonances from it is straightforward and fast. Since a single function operates over a very large frequency range, the method covers a very large number of structures.

Loops have always been difficult to understand intuitively. They have been perceived as impenetrable because of the complicated Bessel formulation of the classical derivation. Our method of transforming the derivation to an RLC formulation gives us greater insight into the modal resonances and how they work together to form the full impedance function presented to an incoming source of energy.

\section{ACKNOWLEDGMENTS}

The corresponding author wishes to thank A. Miroshnichenko from the Nonlinear Physics Centre at the ANU for valuable discussions and C. Jagadish from Electronic Materials Engineering at the ANU for license access to CST's MWS. Thank you also to Pacific Tech. ${ }^{34}$ This work has been partially supported by the Australian Research Council and the Australian Solar Institute.

${ }^{1}$ J. B. Pendry, A. J. Holden, D. J. Robbins, and W. J. Stewart, IEEE Trans. Microwave Theory Tech. 47, 2075 (1999).

${ }^{2}$ A. Locatelli, IEEE Photon. J. 3, 845 (2011).

${ }^{3}$ D. R. Chowdhury, R. Singh, M. Reiten, H.-T. Chen, A. J. Taylor, J. F. O'Hara, and A. K. Azad, Opt. Express 19, 15817 (2011).

${ }^{4}$ A. Ahmadi and H. Mosallaei, Opt. Lett. 35, 3706 (2010).

${ }^{5}$ V. Delgado, O. Sydoruk, E. Tatartschuk, R. Marques, M. J. Freire, and L. Jelinek, Metamaterials 3, 57 (2009).

${ }^{6}$ K. Aydin and E. Ozbay, J. Appl. Phys. 101, 024911 (2007).

${ }^{7}$ C. Enkrich, M. Wegener, S. Linden, S. Burger, L. Zschiedrich, F. Schmidt, J. F. Zhou, T. Koschny, and C. M. Soukoulis, Phys. Rev. Lett. 95, 203901 (2005).

${ }^{8}$ W. J. Padilla, D. N. Basov, and D. R. Smith, Mater. Today 9, 28 (2006).

${ }^{9}$ C. Rockstuhl, T. Zentgraf, H. Guo, N. Liu, C. Etrich, I. Loa, K. Syassen, J. Kuhl, F. Lederer, and H. Giessen, Appl. Phys. B 84, 219 (2006).

${ }^{10}$ M. Staffaroni, J. Conway, S. Vedantam, J. Tang, and E. Yablonovitch, Photonics Nanostruct. -Fundam. Appl. 10, 166 (2012).

${ }^{11}$ A. Salandrino, A. Alù, and N. Engheta, J. Opt. Soc. Am. B 24, 3007 (2007).

${ }^{12}$ A. Alu, A. Salandrino, and N. Engheta, J. Opt. Soc. Am. B 24, 8 (2007).

${ }^{13}$ N. Engheta, A. Salandrino, and A. Al, Phys. Rev. Lett. 95, 95504 (2005).

${ }^{14}$ A. Locatelli, C. De Angelis, D. Modotto, S. Boscolo, F. Sacchetto, M. Midrio, A.-D. Capobianco, F. M. Pigozzo, and C. G. Someda, Opt. Express 17, 16792 (2009).

${ }^{15}$ A. Alu and N. Engheta, Phys. Rev. Lett. 101, 43901 (2008).

${ }^{16}$ O. Sydoruk, E. Tatartschuk, E. Shamonina, and L. Solymar, J. Appl. Phys. 105, 14903 (2009).

${ }^{17}$ S. A. Cummer, B. I. Popa, and T. H. Hand, IEEE Trans. Antennas Propag. 56, 127 (2008).

${ }^{18}$ M. Shamonin, J. Appl. Phys. 95, 3778 (2004).

${ }^{19}$ L. Zhou and S. T. Chui, Phys. Rev. B 74, 035419 (2006).

${ }^{20}$ J. E. Storer, "Impedance of the thin wire loop," Tech. Rep. Report \#212 (Cruft Laboratory, Harvard University, 1955), p. 6.

${ }^{21}$ S. Krishnan, L. Le-Wei, L. Mook-Seng, and K. Pang-Shyan, Microwave Opt. Technol. Lett. 34, 377 (2002).

${ }^{22}$ E. Hallen, Nova Actae Regiae Soc.Sci. Ups. Ser. IV 11, 1 (1938).

${ }^{23}$ T. T. Wu, J. Math. Phys. 3, 1301 (1962).

${ }^{24}$ B. A. Rao, IEEE Trans. Antennas Propag. 16(2), 269 (1968).

${ }^{25}$ M. Kanda, IEEE Trans. Electromagn. Compat. EMC-26, 102 (1984).

${ }^{26}$ K. Iizuka, IEEE Trans. Antennas Propag. 13, 7 (1965).

${ }^{27}$ L. RongLin, N. A. Bushyager, J. Laskar, and M. M. Tentzeris, IEEE Trans. Antennas Propag. 53, 3920 (2005).

${ }^{28}$ L. Le-Wei, L. Mook-Seng, K. Pang-Shyan, and Y. Tat-Soon, IEEE Trans. Antennas Propag. 45, 1741 (1997).

${ }^{29} \mathrm{C}$. Balanis, Antenna Theory: Analysis and Design, 3rd ed. (Wiley-Interscience, 2005), p. 245. 
${ }^{30}$ R. W. P. King, "The loop antenna for transmission and reception," in Antenna Theory, Part 1, Inter-University Electronic Series, Vol. 7, 1st ed., edited by R. E. Collin and F. J. Zucker (McGraw-Hill, New York, 1969), Chap. 11, pp. 458-482.

${ }^{31}$ M. Abramowitz and I. Stegun, Handbook of Mathematical Functions with Formulas, Graphs and Mathematical Tables, Applied Mathematics Series, Vol. 55 (US. Government Printing Office, WDC, 1964), p. 1046. See particularly page 275, Eqs. (9.6.12) and (9.6.13).
${ }^{32}$ See www.cst.com for Computer Simulation Technology AG, Microwave Studio, 2012, Darmstadt, Germany.

${ }^{33}$ MWS requires a frequency band for the test; we chose $10 \mathrm{GHz}$ to $260 \mathrm{GHz}$. This is irrelevant for the theory, since Maxwell's equations scale with frequency and PEC materials are assumed throughout.

${ }^{34}$ See www.pacifict.com. for use of the Graphing Calculator, Pacific Tech. Berkeley, CA. 2012. 